\title{
Cutting the cord
}

\author{
Advances in flexible electronics and soft robotics are yielding wireless devices and untethered machines of \\ increasing sophistication.
}

lectronic devices are typically made

- from hard, rigid materials. Often this isn't a problem, as electronic components and circuits operate in structured environments such as within laptops or smartphones. Robots are also typically made from hard, rigid materials. This isn't necessarily a problem either, as the machines also operate in structured environments such as within manufacturing facilities, carrying out a single programmed task with limited contact with people. But as electronics and robotics become increasingly integrated into everyday life, what is required of these systems is changing and researchers are exploring the potential of soft, flexible materials.

For electronics, systems that offer mechanical flexibility are of particular interest in the construction of wearable and implantable devices, where contact with skin or soft tissue can be key. These devices are often made of thin-film transistors that are mounted on flexible plastic substrates, and a variety of relatively intricate circuits have already been built with such methods ${ }^{1}$. In recent years, wearable sensors and artificial skins have been at the forefront of developments, and devices with an array of sensing capabilities have been reported. Wireless sweat sensors have, for example, been assembled that can monitor a range of physiological biomarkers, sending the data in real time to a smartphone ${ }^{2}$. Alternatively, stretchable devices have been fabricated that do not require any batteries and can measure the optical properties of the skin, while also wirelessly delivering the data to a smartphone . $^{3}$

The capabilities of flexible electronic devices continue to expand, as highlighted in this issue of Nature Electronics. First, Feng Miao and colleagues show that memristors with good thermal stability, which is lacking in traditional memristors, can be fabricated through the stacking of different two-dimensional materials. Notably, they also show that these heterostructure devices can be fabricated on a polyimide substrate, creating flexible memristors that offer both high thermal stability and good flexibility. Furthermore, Daichi Chiba and colleagues report that flexible giant magnetoresistive devices can be used to detect the direction of strain in a material. These flexible spintronic devices are fabricated on a flexible polyethylene naphthalate substrate, and have a trilayer structure consisting of two ferromagnetic layers separated by a non-magnetic spacer layer - one of the ferromagnetic layers is a strain-sensitive free layer, the other a strain-insensitive pinned layer.

For robotics, the building of systems made from soft, deformable materials could allow machines to better adapt to their surroundings and to improve the way they interact with humans. A soft robot could, for example, squeeze into tight spaces, or grasp and manipulate delicate objects. A variety of mobile soft robots have been created so far, including ones that can walk (Fig. 1), jump, climb and swim. However, soft robots often depend on electrical or pneumatic tethers. Ideally, all of the components of a robot including those required for processing, actuation and power - would be contained within its own structure. In a Review Article in this issue, Steven Rich, Robert Wood and Carmel Majidi examine what it takes to cut this cord and build untethered soft robots.

Most soft robotic systems rely on electronics for on-board processing, to control soft actuators, and to sense environmental stimuli. Wider developments in flexible electronics are thus likely to be of value in the ongoing evolution of soft robotics, and further integration of the two fields is certainly likely in the future ${ }^{4}$. In their review, Majidi and colleagues explore recent advances in actuation and electronics, and the systems-level integration required to create functional untethered soft robots. They highlight a number of examples of fully untethered systems - which include robotic caterpillars, fish, rays and octopuses - but also point out that "there still remains considerable work to be done before reliable, untethered functionality in soft robotics can be achieved".

They do, however, go on to carefully consider the challenges involved in engineering robots that could be of practical value. "Improvements in hardware for actuation, sensing and on-board electronics

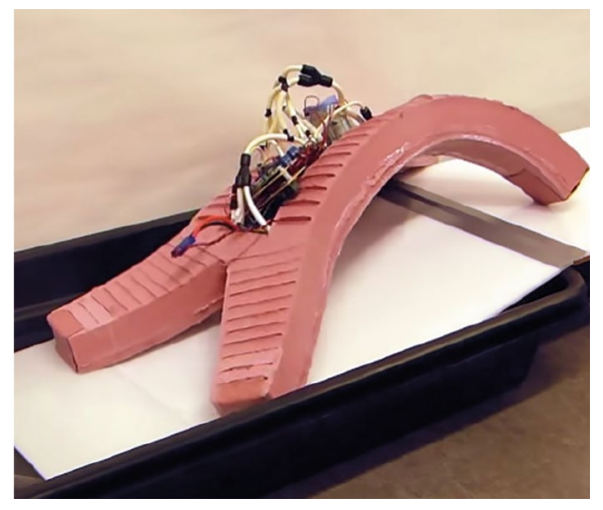

Fig. 1 An untethered soft robot that can walk through difficult terrains. The robot is $65 \mathrm{~cm}$ long and is made of silicone. Credit: Reproduced from ref. ${ }^{5}$, Mary Ann Liebert.

will likely drive the development of these systems. However, further progress will also increasingly depend on advancements in feedback control, machine intelligence and computational modelling." The authors consider in detail three general areas of development: materials improvement, materials integration, and intelligence and autonomy.

Living organisms have been a frequent source of inspiration in the development of flexible electronics and soft robotics, be it skin-inspired wearable devices or fishinspired swimming robots. And it is the relationship between living and artificial systems that is likely to see the greatest benefit from further progress. As Majidi and colleagues note: "In the near future, advances in the field will begin to yield new consumer, healthcare and industrial technologies that will have a transformative impact on how we interact with machines and electronics."

Published online: 8 February 2018 https://doi.org/10.1038/s41928-018-0037-9

\footnotetext{
References

1. Myny, K. Nat. Electron. 1, 30-39 (2018)

2. Gao, W. et al. Nature 529, 509-514 (2016).

3. Kim, J. et al. Sci. Adv. 2, e1600418 (2016).

4. Rus, D. \& Tolley, M. T. Nature 521, 467-475 (2015).

5. Tolley, M. T. et al. Soft Robot. 1, 213-223 (2014).
} 